\title{
Prevalência das lesões cervicais não cariosas em adultos jovens e hipersensibilidade dentinária cervical na Faculdade de Odontologia do Recife
}

Prevalence of non-carious cervical injuries in young adults and cervical dentin hypersesitivity at the School of Dentistry of Recife

Prevalencia de lesiones cervicales no cariosas en adultos jóvenes e hipersensibilidade dentinal cervical en la Faculdade de Odontología de Recife

\section{Resumo}

O objetivo dessa pesquisa foi estimar a prevalência das lesões cervicais não cariosas (LCNC) e hipersensibilidade dentinária cervical (HDC) de adultos jovens da população do Recife, apontando o percentual das LCNC's e HDC nos indivíduos pesquisados, perfil sócio-econômico e demográfico e destacar o diagnóstico situacional das LCNC's e HDC para a população estudada. Foram examinados 60 pacientes na clínica escola da Faculdade Odontologia do RecifeFOR, utilizando um questionário como instrumento de coleta de dados. O exame clínico foi utilizado para verificar a presença de LCNC's, além da Hipersensibilidade Dentinária Cervical. Os dados foram analisados descritivamente por meio de frequências absolutas e percentuais para as variáveis categóricas e das medidas: média, desvio padrão e mediana da variável idade e os resultados foram apresentados em tabelas contendo os valores obtidos na pesquisa dos questionários. Dos 60 pacientes, a maioria $(55,0 \%)$ afirmaram que costumavam ingerir alimentos ácidos e que tal ocorrência se deve provavelmente a uma mudança de hábitos alimentares e estilo de vida. Embora a prevalência de LCNC esteja aumentando com a idade, no estudo presente, não foi possível observar essa prevalência, pois apenas 5,0\% dos 60 pacientes pesquisados apresentaram LCNC e 13,3\% HD.

Palavras-chave: Estudos de prevalência; Hipersensilidade; Causalidade multifatorial.

\footnotetext{
Abstract

The objective of this research was to estimate the prevalence of non-carious cervical lesions (NCCL) and cervical dentinal hypersensitivity (HDC) in young adults from the population of Recife, pointing out the percentage of LCNC's and HDC in the individuals surveyed, socioeconomic and demographic profile and highlighting the situational diagnosis of NCCL 's and HDC for the studied population. Sixty patients were examined at the teaching clinic of Faculdade de Odontologia do Recife-FOR, using a questionnaire as an instrument for data collection.Clinical examination was used to verify the presence of lesions on the buccal surface of premolars and first molars of all quadrants, in addition to
} 
Cervical Dentin Hypersensitivity. Clinical examination was used to verify the presence of NCCL's, in addition to Cervical Dentin Hypersensitivity. Data were descriptively analyzed using absolute frequencies and percentages for categorical variables and measures: mean, standard deviation and median for the age variable, and the results were presented in tables containing the values obtained in the survey of the questionnaires. Of the 60 patients, the majority $(55.0 \%)$ stated that they used to eat acidic foods and that this occurrence is probably due to a change in eating habits and lifestyle. Although the prevalence of NCCL is increasing with age, in the present study, it was not possible to observe this prevalence, as only $5.0 \%$ of the 60 patients surveyed had LCNC and $13.3 \%$ had HD.

Keywords: Prevalent studies; Hypersensitivity; Multifactorial causality.

\section{Resumen}

El objetivo de esta investigación fue estimar la prevalencia de lesiones cervicales no cariosas e hipersensibilidad dentinaria cervical de adultos jóvenes en la población de Recife, indicando el porcentaje de LCNC y HDC en los individuos estudiados, perfil socioeconómico y demográfico y destacar el diagnóstico situacional de LCNC y HDC para la población estudiada. Examinamos a 60 pacientes en la clínica escolar de la Escuela de Odontología de Recife-FOR, utilizando un cuestionario como instrumento de recolección de datos. El examen clínico se utilizó para verificar la presencia de LCNC, además de hipersensibilidad dentinaria cervical. Los datos fueron analizados descriptivamente mediante frecuencias absolutas y porcentajes para variables categóricas y mediciones: media, desviación estándar y mediana de la variable edad y los resultados se presentaron en tablas que contienen los valores obtenidos en la encuesta de los cuestionarios. De los 60 pacientes, la mayoría $(55,0 \%)$ declaró que solía comer alimentos ácidos y que esta ocurrencia probablemente se deba a un cambio en los hábitos alimenticios y el estilo de vida. Aunque la prevalencia de LCNC está aumentando con la edad, en el presente estudio, no fue posible observar esta prevalencia, porque solo el $5,0 \%$ de los 60 pacientes encuestados tenían LCNC y el 13,3\% HD.

Palabras clave: Estudios de prevalencia; Hipersensilidad; Causalidad multifactorial.

\section{Introdução}

As lesões cervicais não cariosas (LCNC) e hipersensibilidade dentinária cervical (HDC) são doenças multifatoriais formadas livres das ações de microorganismos, de cárie ou processos inflamatórios, e se apresentam em várias morfologias, como sulcos rasos, lesões extensas supra e/ou subgengivais. Diversos estudos demonstram a relação direta das LCNCs e HDC com o estilo de vida do paciente destacando a biocorrosão dental (Soares et al, 2014; Sugita et al, 2017; Zeola et al., 2018).

Atualmente, as LCNCs são consideradas de etiologia multifatorial. Vários mecanismos, podem estar associados a sua origem e progressão. Variam desde pequenas ranhuras até amplas cavidades sem processo carioso. Podem se apresentar em forma de cunha e disco, angulado e com bordas bem definidas e ainda em forma de "U" ou "V" com ângulos bem definidos. As causas mais frequentes são abrasão, erosão e trauma occlusal (Oliveira et al., 2010; Costa et al, 2018, Borcic et al, 2004).

O terço cervical é considerado mais suscetível para ação e desgaste pelos fatores etiológicos das LCNCs, devido a morfologia das estruturas dos tecidos dentários que apresenta o esmalte bastante fino e o cemento e a dentina não são muito resistentes. O enfraquecimento do esmalte e dentina cervical, ao nível da junção amelo-dentinária (JAD), pela tensão com a ação conjunta de fatores corrosivos e de fricção, também afetam o tecido periodontal e podem estar relacionados com a recessão gengival (El-Marakby et al, 2017, Soares et al, 2017).

O declínio na prevalência de cárie dentária na população mundial que vem ocorrendo ao longo dos últimos dez anos, tem sido acompanhado por um aumento na incidência de lesões não cariosas, que levam a uma perda irreversível da estrutura dentária. A presença dessas lesões pode ocasionar a hipersensibilidade dentinária em que um indivíduo se revela com dor após a exposição dos túbulos dentinários em virtude do desgaste do esmalte e cemento (Teixeira et al, 2018; Souza et al, 2017; Machado et al, 2018).

A prevalência de LCNC tem aumentado com a idade, variando de 5 a $85 \%$ nas dentições atuais e é mais comumente encontrada nos pré-molares e molares. Os dados na literatura são altamente discrepantes e determinados pelos critérios de defeito da morfologia da LCNC. Essa alta variação aponta a dificuldade de definir o que constitui um mecanismo etiológico único para LCNC. Torna-se difícil chegar a um valor preciso de prevalência para todas as populações porque fatores como idade e grupo 
étnico criam grandes variações em números. É provável que as HDCs sejam originadas subgengivalmente e que haja uma correlação direta com a formação das LCNC incipientes (Teixeira et al, 2018)

A relação da LCNC com hipersensibilidade dentinária (HD), dá-se devido à exposição dos túbulos dentinários. Os prémolares são os mais afetados pela maior exposição de dentina por causa da sua anatomia e, consequentemente, são mais afetados pela HD. Acomete muitos pacientes, podem ser sintomáticas ou assintomáticas e o tratamento consiste na remoção deste fator para aliviar a dor. Uma alternativa é a restauração com a resina composta, visto que a origem da perda mineral será cessada (Senna et al, 2012).

Para o correto e precoce diagnóstico, é necessário que o Cirurgião-Dentista (CD) atente para o fato de que os desgastes dentários, comumente apresentam etiologia multifatorial, sendo necessária uma minuciosa anamnese e exame clínico apurado para iniciar a terapêutica. Durante esses procedimentos, o profissional deverá identificar possíveis fatores de interferência oclusal e presença de hábitos parafuncionais para, então, traçar um plano de tratamento que poderá englobar orientação dietética, controle psicoemocional, em conjunto com outros profissionais para reabilitar a saúde do paciente, além de etapas restauradora, oclusal e endodôntica (Dotto et al, 2008).

Do mesmo modo que a etiologia das LCNCs é multifatorial, o tratamento também deve ser multidisciplinar, englobando várias áreas da odontologia (Mendes-Júnior et al, 2021). Isso permite que o tratamento concentre-se na causa do problema e não nos sintomas. Alguns tratamentos são mais indicados como, ajuste oclusal, no qual o objetivo principal do procedimento é determinar na boca os dentes que estão em prematuridade cêntrica, pois são mais frequentemente associados à deflexão em inclinações no plano sargital. Esse ajuste é proposto como alternativa de prevenir o início e progressão dessas lesões. Já para HDC são indicados agentes dessensibilizantes e laserterapia (Brandini et al, 2012; Modanese et al, 2018; Soares et al, 2014; Miranda et al., 2021).

Diante do exposto, é notável que as LCNC's ocasionam problemas estéticos e funcionais, sendo de grande importância registrar as variáveis bucais, comportamentais e sistêmicas comuns aos pacientes portadores destas lesões. Visto que a prevalência das LNCN em adultos jovens está aumentando, o objetivo dessa pesquisa foi estimar a prevalência das lesões cervicais não cariosas e hipersensibilidade dentinária cervical de adultos jovens da população do Recife, apontando o percentual das LCNC's e HDC nos indivíduos pesquisados, perfil sócio-econômico e demográfico e destacar o diagnóstico situacional das LCNC's e HDC para a população estudada. Onde acredita-se que com o atual estilo de vida dos jovens adultos, o índice de LCNC e HD vêm aumentando.

\section{Metodologia}

Tratou-se de uma pesquisa descritiva, exploratória, de campo, transversal com abordagem quantitativa, por se tratar de um estudo que apresenta informações precisas e interpretáveis. Os participantes da pesquisa somente submeteram-se aos procedimentos de coleta de dados, após a assinatura do termo de consentimento livre e esclarecido. Este estudo foi aprovado pelo Comitê de Ética em Pesquisa da Universidade Estadual de Pernambuco (HUOC/PROCAPE) pelo parecer de número 4.675.511, seguindo as diretrizes e normas estabelecidas pela Resolução CNS n 466 de 2012 e na Norma Operacional no 001 de 2013 do CNS. Sendo riscos contidos no presente estudo são os inerentes aos projetos desta natureza. Uma vez realizados com a observância das normas preconizadas pela Comissão Nacional de Ética em Pesquisa em Seres Humanos, garantindo assim o sigilo ético, podendo desta forma fazer a afirmação que os riscos são mínimos.

A amostra foi por conveniência e aleatória formada por 60 pacientes, que foram atendidos nas clínicas da Faculdade de Odontologia do Recife (FOR) no período de agosto a novembro de 2021. Os pacientes apresentavam idade mínima de 19 anos e máxima de 30 anos de ambos os sexos e aceitaram participar da pesquisa mediante assinatura do TCLE - Termo de Consentimento Livre e Esclarecido. 
Foi utilizado um questionário, como instrumento de coleta de dados, cujo preenchimento foi efetuado por um pesquisador calibrado, baseado na prevalência de lesões cervicais não cariosas em uma população adulta jovem sendo 8 questões fechadas de múltipla escolha com perguntas relacionadas aos hábitos alimentares, parafuncionais, sensibilidade, estilo de vida, entre outros e 1 questão aberta relacionada a queixa principal do paciente. Após o preenchimento do questionário, cada indivíduo foi submetido a um exame clínico, realizado por um examinador, para verificar a presença da LCNC, desgastes oclusais dos dentes, exposição de dentina, recessão gengival e erosão.

O exame foi visual e tátil utilizando uma paleta de madeira, onde foram analisadas a presença ou não de guia canina, interferência oclusal em dente anterior e posterior, e presença de desgastes oclusais ocasionados por hábitos parafuncionais. Para a análise da Hipersensibilidade dentinária foi utilizado jato de ar, com distância aproximada de $2 \mathrm{~mm}$ do substrato dental, nos dentes que apresentavam LCNC e recessão gengival, para que o próprio paciente relatasse o grau da sensibilidade, que variava entre 1 e 10, e fosse assim preenchido o questionário. A presença de qualquer irregularidade foi considerada lesão, mesmo se esta estivesse localizada na JCE. ${ }^{16}$ Foram consideradas lesões detectadas visualmente e tatilmente; lesões restauradas não foram avaliadas. Como critério de exclusão, indivíduos portadores de aparelho ortodôntico e pacientes com agenesia ou ausência dos elementos avaliados não participaram da pesquisa.

Os dados foram analisados descritivamente por meio de frequências absolutas e percentuais para as variáveis categóricas e das medidas: média, desvio padrão e mediana da variável idade e os resultados foram apresentados em tabelas contendo os valores obtidos na pesquisa dos questionários. Os dados foram digitados na planilha EXCEL e o programa utilizado para obtenção dos cálculos estatísticos foi o IMB SPSS na versão 25.

Os benefícios esperados com o desenvolvimento do presente estudo, consiste em orientar a mudança de hábitos dos adultos jovens, como alimentação, hábitos de higiene, visto que os indivíduos estão permanecendo por mais tempo com dentição permanente na cavidade oral. Para isso, cuidados precisam ser tomados, para ocorrer a redução da prevalência de Lesões Cervicais não Cariosas e Hipersensibilidade Dentinária, destacando os cuidados na rotina que as pessoas precisam tomar.

\section{Resultados e Discussão}

A idade dos pacientes pesquisados variou de 19 a 30 anos, teve média de 24,35 anos, desvio padrão de 2,64 anos e mediana igual a 24,00 anos. Nos Gráficos 1 e 2 se apresentam as características da amostra pesquisada onde pode ser verificado que: um pouco mais da metade $(55,0 \%)$ era do sexo feminino; a maioria $(81,7 \%)$ era procedente do Recife e o restante era do interior $(15,0 \%)$ ou de outros dois munícipios da Região Metropolitana.

Gráfico 1 - Frequência percentual do sexo dos pacientes pesquisados.

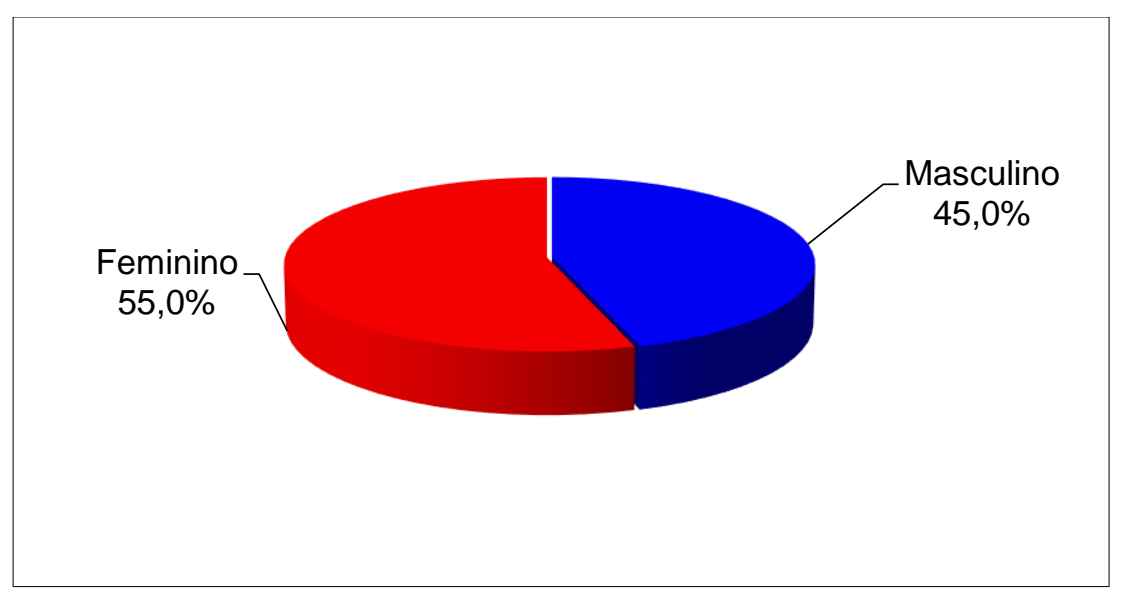

Fonte: Autores. 
Gráfico 2 - Frequência percentual da procedência dos pacientes pesquisados.

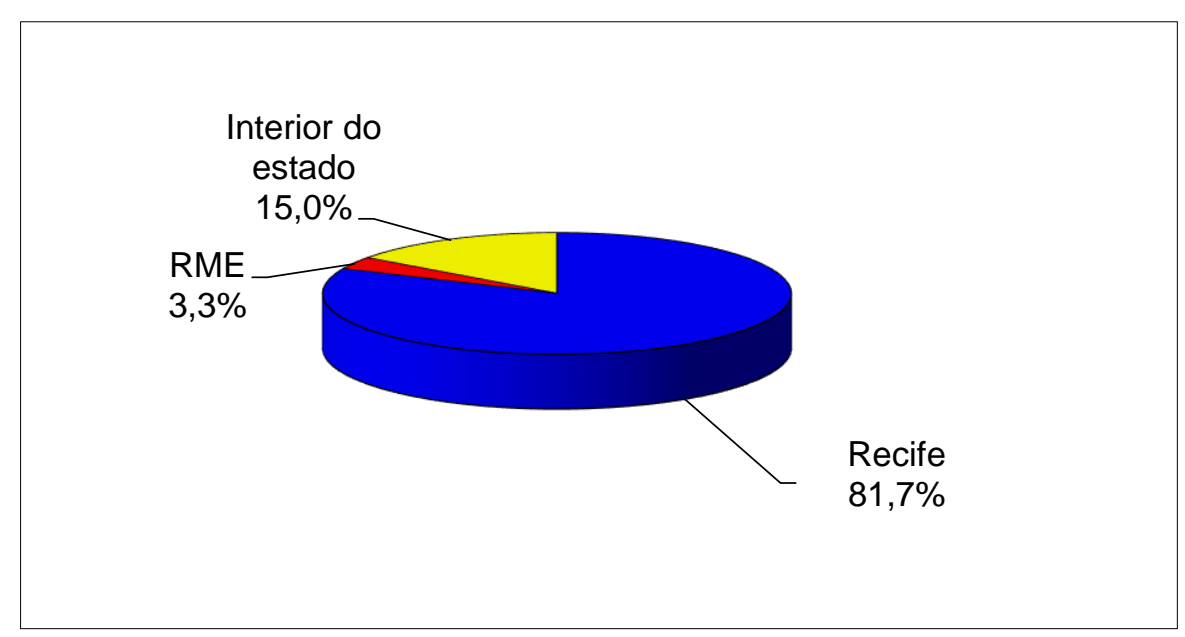

Fonte: Autores.

Dos resultados contidos no Gráfico 3 e 4 destaca-se que nas clínicas de dentística foi onde houve a queixa principal mais prevalente, com 43,3\% da amostra, seguida das queixas exame de rotina $(16,7 \%)$, periodontia $(13,3 \%)$ e endodontia $(10,0 \%)$ e as outras duas queixas listadas variaram de $1,7 \%$ a $6,7 \%$; das queixas da dentística as duas causas mais prevalentes foram: dentes amarelados $(18,3 \%)$ e dentes fraturados (10,0\%); dos 8 pacientes que tiveram queixas da periodontia, 6 tiveram excesso de gengiva e os outros dois gengivite. A maioria $(85,0 \%)$ não teve placa visível.

Gráfico 3 - Frequência percentual da queixa principal dos pacientes.

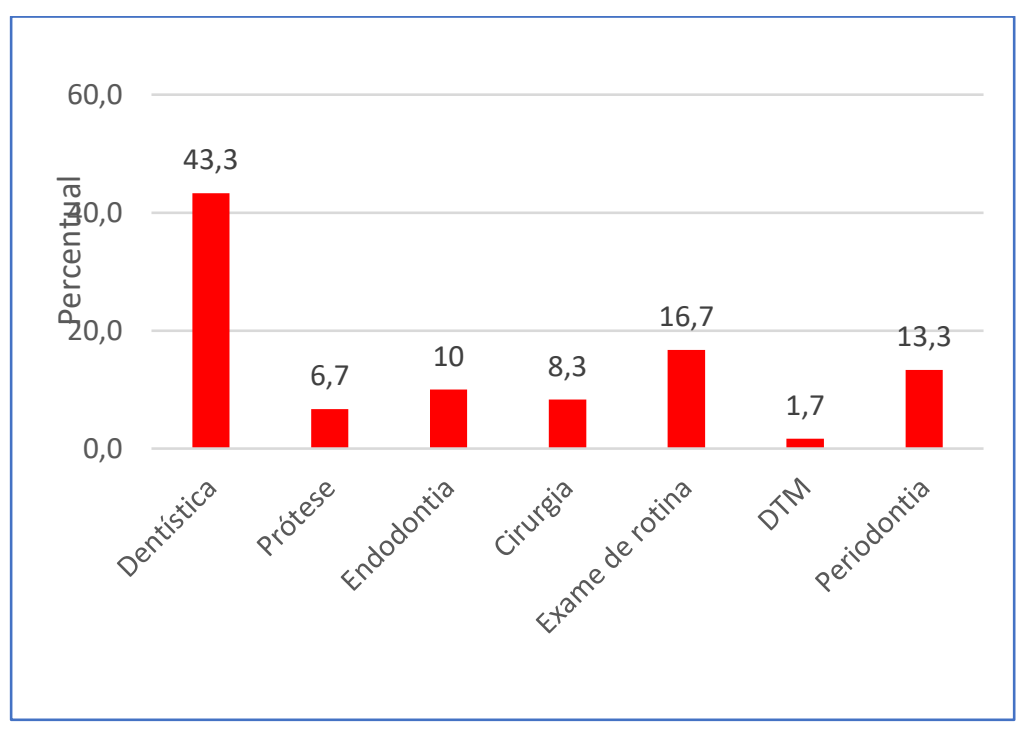

Fonte: Autores. 
Gráfico 4 - Frequência percentual da qualidade da higiene oral.

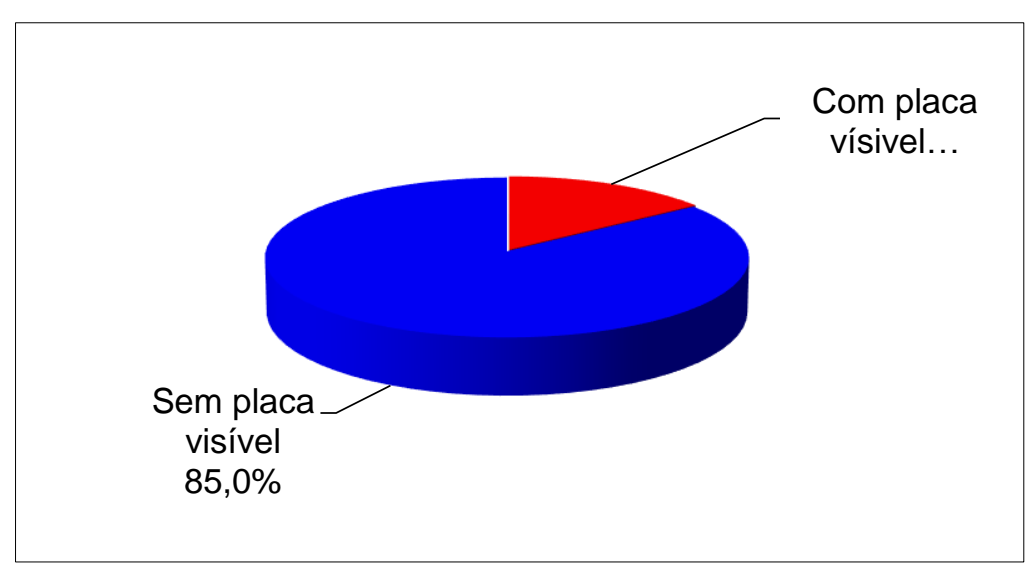

Fonte: Autores.

Dos resultados contidos no gráfico 5 se enfatiza que: um pouco mais da metade $(53,3 \%)$ afirmou que não realizava escovação com força excessiva; a maioria $(55,0 \%)$ afirmou que costumava ingerir alimentos ácidos; exatamente 1/4 $(25,0 \%)$ apresentava alguma doença gástrica; o percentual que afirmou que tinha algum hábito parafuncional foi 30,0\%; a presença de trauma oclusal foi registrado em 13,3\% do grupo e 5,0\% apresentavam lesão cariosa e/ou não cariosa (LCNC); a presença de hipersensibilidade dentinária foi observada em 13,3\% e nestes foram observados sensibilidade em um dente em 5 pacientes e dois dentes nos outros três pacientes. Na tabela a listagem de 11 elementos cada sensibilidade, cada um com frequência unitária.

Outros resultados apurados e não incluídos no gráfico foi que entre os três pacientes com LCNC, cada um tinha um dente com a lesão correspondente a duas LCNC angulada e uma arredondada nos elementos dentários 24, 44 e 46.

Gráfico 5 - Frequência percentual das questões sobre dados clínicos dentários.

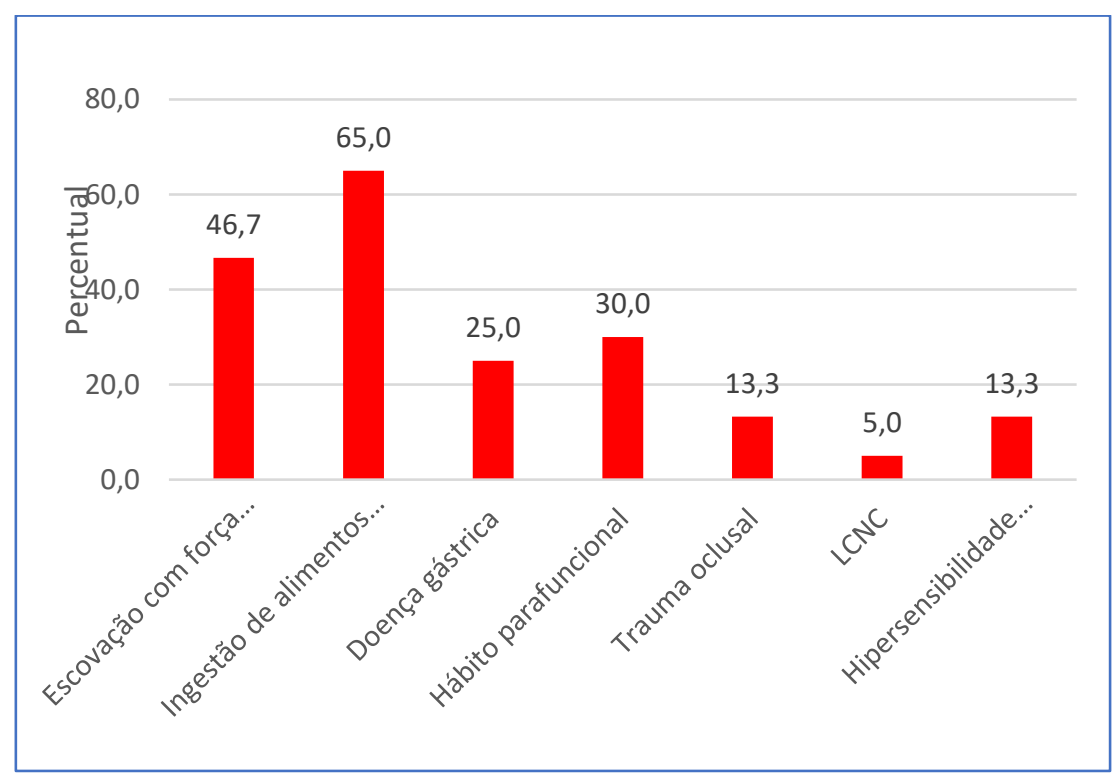

Fonte: Autores.

No presente estudo foi analisado que houve um resultado diferente do que se esperava no começo do estudo, ocorrendo uma baixa prevalência das LCNC'S na população estudada. A amostra de pacientes avaliados apresentou-se uniforme em relação à idade. Foram avaliados 60 pacientes atendidos nas clínicas da FOR com idade média de 24,35 anos. Destes, a maioria (81,7\%) 
era procedente do Recife e o restante era do interior (15,0\%) ou de outros dois municípios da Região Metropolitana. A maioria dos pacientes eram do sexo feminino $55 \%$ e $45 \%$ do sexo masculino.

Das queixas principais, a maior incidência se deu na clínica de Dentística, com a queixa de dentes amarelados 18,3\%. Dos 60 pacientes, a maioria $(55,0 \%)$ afirmaram que costumavam ingerir alimentos ácidos e que tal ocorrência se deve provavelmente a uma mudança de hábitos alimentares e estilo de vida, como também afirmam Sytruzycka et al (2012) em seu estudo sobre Prevalência de lesões erosivas como fatores de risco em uma população jovem adulta na Polônia.

Teixeira, et al. (2018), também afirmam que a exposição repetida ou prolongada dos dentes aos ácidos gástricos leva à dissolução seletiva da superfície dentária, causando perda da estrutura e HD. Porém, embora a prevalência de LCNC esteja aumentando com a idade, no estudo presente, não foi possível observar essa prevalência, pois apenas 5,0\% dos 60 pacientes pesquisados apresentaram LCNC. Valendo ressaltar, como também afirmado por Teixeira, et al. (2018), torna-se difícil chegar a um valor preciso de prevalência para todas as populações porque fatores como idade e grupo étnico criam grandes variações em números.

O estudo também mostrou que 85,0\% não teve placa visível, evidenciando que com o passar dos anos os indivíduos estão tomando mais cuidado com a saúde bucal, na rotina, visto que com a odontologia preventiva, as pessoas estão envelhecendo e permanecendo com os dentes na cavidade oral e isso, associado aos fatores extrínsecos e intrínsecos contribui para uma maior formação de LCNC (Mangueira et al, 2011; Amaral et al, 2012). Outros fatores que evidenciaram tal afirmativa anterior, foram os 53,3\% que não realizaram escovação excessiva, que afirmavam saber que era o certo.

Porém, mesmo sendo comprovado esse aumento de pessoas que estão envelhecendo com os dentes na cavidade oral, muitos dos pacientes entrevistados na clínica mostraram ainda não possuir tempo de exposição suficiente para evidenciar as LCNC e a HD, pois um grupo de 5,0\% apresentavam LCNC, e 13,3\% apresentavam HD (Hipersensibilidade Dentinária), sendo está analisada em pacientes que possuíam as LCNC e pacientes com recessão gengival, onde parte desses estão dentro dos 46,7\% que escovavam com força excessiva.

Exatamente 1/4 (25,0\%) apresentava alguma doença gástrica onde, Teixeira et al (2018) demonstraram que a exposição repetida ou prolongada dos dentes aos ácidos gástricos leva à dissolução seletiva da superfície dentária, causando perda da estrutura e HD. O percentual que apontou entre os pacientes pesquisados algum tipo hábito parafuncional foi 30,0\%, e a presença de trauma oclusal foi registrado em 13,3\%, dados que concordam com Mondanese et al (2018), que afirmaram, que pacientes com bruxismo têm 6 vezes mais chances de apresentar abfração do que pacientes não bruxista.

Não foi possível realizar testes de associação entre as variáveis, idade e sexo com a presença de LCNC e HD, devido ao pouco número de LCNC nos pacientes pesquisados. Entre os três pacientes com LCNC, cada um tinha um dente com a lesão correspondente a duas LCNC angulada e uma arredondada nos elementos dentários 24, 44 e 46. Das limitações encontradas durante o estudo do trabalho, foi a baixa quantidade de pacientes na faixa dos 19 a 30 anos, juntamente com a falta de mais teorias para que fosse possível um estudo mais aprofundado do assunto.

\section{Conclusão}

Conclui-se no presente estudo que a Prevalência das lesões cervicais não cariosas foi de 5,0\% e hipersensibilidade dentinária cervical de adultos jovens de 13,3\% na população atendida na Faculdade de Odontologia do Recife. A maioria dos pacientes foram do sexo feminino, $55 \%$ e $45 \%$ do sexo masculino. Sendo observada dessa forma, maior prevalência de HD, do que de LCNC. No presente estudo, houve um resultado diferente do que se esperava, ocorrendo uma baixa prevalência das LCNC'S na população estudada, sendo necessários mais estudos que abordem esse assunto e busquem identificar essa prevalência. 


\section{Referências}

Amaral, et al (2012). Lesões não cariosas: o desafio do diagnóstico multidisciplinar. Arquivos Int. Otorrinolaringol, São Paulo, v. 16, n. 1

Borcic J., Anic I., Urek MM., Ferreri S. (2004). The prevalence of non-carious cervical lesions in permanent dentition; Journal of Oral Rehabilitation ;p 117123; Croatia; 2004

Brandini D., Pedrini D., Panzarini S.; , Benete I., Travisan C. (2012). Clinical evaluation of the association of noncarious cervical lesions, parafunctional habits, and TMD diagnosis; V.43; p.255-262; 2012

Costa L.S., Alves S.S., Lima D.D., Dietrich L., ,Santos P.C., ,Martins F.M. (2018). Lesão cervical não cariosa e hipersensibilidade dentária; Revista odontológica Bras Central; p.247-251; 2018

Dotto S., Carvalho M., Pagliarin C., Ferreira F., Villa M. (2008). Lesão cervical näo-cariosa por abfração - terapêutica endodôntica. Revista Dentística on line ;2008

El-Marakby A.M., Al-Sabri F.A., Alharbi S.A., Halawani S.M., Yousef M.T.B. (2017). Noncarious Cervical Lesions as Abfraction: Etiology, Diagnostics, and Treatment Modalities of Lesions: A Review Article. Odontologia 7: 438. doi: 10.4172 / 2161-1122.1000438; 2017

Machado C., Fernandes Neto A.J., Vilela A.L., Menezes M., Teixeira D,, Cardoso I. et al (2018). Influência do desequilíbrio oclusal na origem de lesão cervical não cariosa e recessão gengival: analise por elementos finitos;Rev Odontol Bras Central; 27(83): 204-210;2018

Mangueira, et al (2018). Cárie e erosão dentária: uma breve revisão.Odontol. Clín.-Cient., Recife, v. 10, n. 2, 2011. Mendes-Junior, T. E., Pinheiro, J. C. ., Silva, M. D., Ribeiro, J. G. R., \& Mendes, T. E. . (2021). The importance of transdisciplinary treatment for non-carious cervical lesions and dental hypersensitivity and the influence on quality of life: case report. Research, Society and Development, 10(14), e526101422002. https://doi.org/10.33448/rsd-v10i14.22002

Miranda, D. R. ., Pereira, C. S. ., \& Reis, L. Q. C. . (2021). Tratamento da Hipersensibilidade dentinária nas lesões cervicais não cariosas: revisão da literatura . Research, Society and Development, 10(15), e86101522703. https://doi.org/10.33448/rsd-v10i15.22703

Modanese D.; Canevese V.; Alessandretti R.; Spazzin A.; ,Radaelli M (2018). No-carious abfraction cervical lesions: prevalence and relationship with sleep bruxism; Journal of oral investigation; Vol.7; p.22-32; Passo Fundo; 2018

Oliveira A., Damacesna N., Souza C., (2010). Analise clinica de pacientes portadores de lesões cervicais não cariosas e sua relação com os habitos; Revista sulbrasileira de odontologia; Aracaju; 2010

Senna P., Del Bel Cury A., Rosing C. (2012). Non-carious cervical lesions nos occlusion: A systematic review of clinical studies; Journal of oral rehabilitation; p.2-11; Porto Alegre; 2012

Soares P.V.; Tolentino A.B.; Machado A.C.; Dias R.B.; Coto N.P. (2014); Odontologia do esporte: uma perspectiva de futuro; Odontologia esportiva; Rev Bras Educ Fís Esporte, (São Paulo) Abr-Jun; 28 (2): 351-58 • 353; 2014

Soares P.V., Zeola L.F., Spiri P.H.R., Machado A.C., Pereira F.A., Pereira A.G. et al. (2014). Lesões cervicais não cariosas e hipersensibilidade dentaria: protocolos reabilitadores e estéticos; pro-odonto estética; V.2;p 43-73; Porto Alegre; 2014

Soares P.V. ,Zeola L.F. „Spiri P.H.R. ,Machado A.C. ,Pereira F.A. ,Pereira A.G. (2017). et al; Lesões cervicais não cariosas e hipersensibilidade dentariaconsiderações clinico-cientificas; Porto alegre;2017

Souza P., Machado A., Pereira A., Texeira R., Espinhola F., Soares P.V. (2017). The dentin chemical degradation and saliva roles on Noncarious Cervical Lesions - literature review;Journal of Dental Science;P.199-203;2017

Sugita L., Nakashima S., Ikeda A., Burrow M.F., Nikaido T., Kubo S. et al (2017). A pilot study to assess the morphology and progression of non-carious cervical lesions; Journal of Dentistry; P.51-56; Japão; 2017

Struzycka I., Lussi A,, Kapala A.B., Rusyan E., (2012) Prevalence of erosive lesions with respectto risk factors in a young adult population in Poland- a crosssectional study; Clin Oral invest; 2012

Teixeira D., Zeola L.F., Machado A.C., Gomes R.R., Souza P.G., Mendes D. et al (2018). Relationship between noncarious cervical lesions, cervical dentin hypersensitivity, gingival recession, and associated risk factors: A crosssectional study; Jornal of Dentristy;2018

Zeola L., Soares P.V., Cruz J. (2018). Prevalência de hipersensibilidade dentária: revisão sistemática e meta-análise; Journal of Dentistry; Uberlandia; 2018 